\title{
Call us nurse champions: Lessons learned in strategic partnership with nurses in clinical research
}

\author{
Rebecca Koszalinski*1, Theresa Day ${ }^{2}$, Clay Kyle $^{3}$ \\ ${ }^{1}$ The University of Oklahoma Health Sciences Center, The Fran and Earl Ziegler College of Nursing, United States \\ ${ }^{2}$ Trauma Surgical Intensive Care Unit, University of Tennessee Medical Center Knoxville, United States \\ ${ }^{3}$ Progressive Care Unit, University of Tennessee Medical Center Knoxville, United States
}

Received: July 12, 2020

DOI: $10.5430 / \mathrm{cns} . v 8 \mathrm{n} 4 \mathrm{p} 13$
Accepted: August 26, 2020

Online Published: September 17, 2020

URL: https://doi.org/10.5430/cns.v8n4p13

\begin{abstract}
This manuscript reports the perspectives of nurse managers who participated in a study at a regional medical center. The information is reported through verbatim comments that emerged through a discussion of "lessons learned". Nursing comments are organized as the conversation flowed. The nurse managers that participated in a recently concluded study were most concerned about how any research study will affect their patients and how nurse workflow may be interrupted. Pre-established meeting times (huddles) worked best for education and training. Further, they suggested incentives may be effective; however, recognition as nurse champions and inclusion of nurse voice are preferred.
\end{abstract}

Key Words: Clinical research, Nurse champion, Nurse leader, Collaboration, Research; nurse, Team research, Intensive care, Critical care

\section{INTRODUCTION}

Imagine that an affiliated university researcher briskly walks onto a nursing unit and locates the nurse manager. The researcher pointedly explains that the hospital institutional review board approved a study, but while the previous nurse manager was aware of the study, that person has since moved on to another opportunity. The researcher, not knowing this information, proceeds to instruct the nurse manager about the nurses' responsibilities in the study. As call light indicators incessantly beep and nurses rush through the nurses' station and then back out to patients, the nurse manager stands transfixed in silent dismay. It is doubtful, in this context, that the study will smoothly progress and will result in good outcomes. There is no evidence of collaboration or communication in this vignette.
The key to successful clinical research is clear and consistent communication and collaboration. ${ }^{[1]}$ Lack of collaboration and poor communication overall result in disjointed and ineffective processes. ${ }^{[2]}$ We conducted a study at a local university-affiliated, magnet status hospital to learn if the use of communication technology could affect patient outcomes of symptoms of anxiety and depression. While the results of the study are published elsewhere, ${ }^{[3,4]}$ the purpose of this study report is to discuss our lessons learned in the context of clinical collaboration.

\section{LITERATURE REVIEW}

The concept of collaboration in research (academic or clinical) infers equal partnership. Further, collaboration suggests at least a level of democratization of decision-making that transcends the idea of teamwork. That is, there is intended

\footnotetext{
* Correspondence: Rebecca Koszalinski; Email: Rebecca-Koszalinski@ ouhsc.edu; Address: Fran and Earl Ziegler College of Nursing, University of Oklahoma Health Sciences Center, 1100 N Stonewall Ave, Oklahoma City, OK 73117, United States.
} 
parity among the involved parties. Unfortunately, this parity "belies reality, particularly in Western jurisdictions, where the medical profession has exercised sovereign power over other health professions" $(\mathrm{p} 3,482) .{ }^{[5]}$ The researchers noted in researching clinical protocols that nurses were identified only as assignees or subjects in studies and further argued that many protocols constrain collaboration because they diminish nursing voice. ${ }^{[5]}$ To sum their assertions, collaboration may be restricted because said protocols "create the expectation that nurses work alone across the spectrum of protocols" (p3,482). ${ }^{[5]}$

Therefore, we could consider the concept of partnership in the context of clinical research and recognize the voice of clinical nurses should be heard when nurses participate in any aspect of research. After all, "When collaborating or working as part of a team, an assumption can be made that the unique expertise of individuals, utilized collectively, will improve outcomes, meaning the whole is greater than the sum of the parts" (p120). ${ }^{[6]}$ Further, consider that magnet status hospitals are expected to educate their nurses about research and provide time to participate in research; but realistically, research processes are not so easily executed. Often, it is unfeasible to hire a nurse researcher, which means clinical nurses must be developed internally and are externally sought to "promote, sustain, and evolve nursing research efforts" (p600). ${ }^{[7]}$ Nurses provide valuable information and insight and are integral members of the research team. To that end, we met one-year post conclusion of our study in a local magnet status hospital (2 nurse managers) and then threemonths later with additional nurse managers ${ }^{[3]}$ via phone and video call, per COVID19 precautions, to talk about a previously conducted study and identify lessons learned. What we learned is that communication and collaboration lead to favorable nursing participation experiences.

\section{THE STUDY AND SETTING}

The primary study was a randomized control trial set in an academic magnet status teaching hospital in rural Appalachia. A communication application (Speak for Myself; Speak for Myself-Voice) was offered to patients who met inclusion criteria in five intensive care units. For this query of lessons learned, we informally met for an hour to discuss the study, which was followed by second 45-minute conversation via telephone and video call, per COVID19 social distancing precautions. Although we did not perform qualitative analyses, verbatim comments in this paper are organized as the conversation flowed and thoughts emerged.

\section{HUMAN PROTECTION}

The study was approved by a Graduate School of Medicine Institutional Review Board (IRB) (\#4256).

\section{TWO SIGNIFICANT QUESTIONS THAT NURSES PONDER}

When approached by researchers, clinical nurses generally think of two significant questions. First, they would like to know if the proposed intervention(s) specifically meets the needs of the intended patient population and, second, they would like to know how their workflow will be affected.

"The first thing is to put my patient in the middle to see if it will impact my patient. How will the outcome impact my staff, and what will it do to workflow?"

"I also want to know what the benefit is going to be to the patient and to the staff because I hate to be selfish in that way, but I want to know if my patient will benefit and will the staff because I am always looking to improve."

Nurses are more likely to participate in research assignments if they can detect perceptible benefits for the patient or if the study results in improved workflow. If the workflow is likely to remain undisturbed, then clinical nurses may commit to the study and fully participate. Conversely, if the clinical nurses are required to set time aside from their usual schedule, it is unlikely the nurses will fully participate. Also, it may be beneficial for nurse managers to review any questionnaire or survey to better understand time requirements.

"For my unit, unless someone else is paying them or they are on their day off, there is not an hour built into their shift to be able to do anything. That's why I said I need to understand how it is going to impact the nursing workflow and does it benefit the patient and will that outweigh the interruption of the nursing workflow because right now, virtually every minute of their day is committed to some sort of task to meet the needs of that patient. To take a full hour out of anything, it's hit or miss. If one of my patients go to surgery and they [nurses] have some extra time, then absolutely, then I could commit that time to helping with the research but, if instead, my patient codes, then I am not going to have that hour to commit to anything other than keeping that patient alive."

"The buck stops with the nurse. Um, when there is not a CNA, the nurse must do the extra work when there's nobody to pass the tree that gets shoved to the nurses. So, it's like we keep asking them to do one more thing, one more thing, one more thing."

"For my nurses, they don't really mind a little disruption or a little extra work. They're ok with a little extra work as long they can see that the outcome for the patients is going to be better, um, uh, if it's. . if it's a project that you don't really know that there's going to be a better outcome or you don't know that there's really a need then it's much more difficult to disrupt their workflow."

ISSN 2324-7940 E-ISSN 2324-7959 
"I mean every day we are looking at ways to be more efficient to give our nurses more time with the patient, so everything we do has to take time away so definitely they don't have an hour."

However, if a researcher brings a research study that does not require time away from the patient but they are asked only to be aware and assist with identification of potential study participants, followed by contact to the research team, then clinical nurses are more willing to participate.

\section{WHAT WE LEARNED}

\subsection{Education is crucial: Huddles worked}

The nurses were asked about huddle at shift change: Was teaching at huddle effective for all of you?

"For us it was beneficial. . . because it helped with communication between patient and the nurse, patient and provider, and also the education portion of it was easy. It wasn't anything extra. It just went right into the huddle. And then we were able to use the device with the patient and then being able to call you to do most of the, y'know, most of the background work was beneficial."

"For me, I would say yes because we were able to do the education in a format that we already use. We didn't have to implement anything, so we were able to take time out for something we do every day anyway and educate in that way. I think it would have been nice if we could have added some champions to the project because we do have people that do above and beyond. You know, spend a little extra time on their days off or do guest projects whatever. They would participate in that way and donate some of their time, so that's something to consider in the future."

"It is a time we already have set aside for new information and for reinforcing current information, so huddle time is time we already have set aside. So, the time worked very well for us. If we had champions for a research project and someone says I am passionate, I am committed, this is going to benefit your patient or fellow co-workers or whatever the benefit may be; then what the team leaders can do, at least in my unit, is to take that into consideration when they are making assignments so if I know that nurse A is committed to this project and when there is time, they are going to devote extra time to helping, then I am going to give Nurse A an assignment that may allow for a little wiggle room today. I am not going to give Nurse A the patient that I know will require undivided attention. I am going to give Nurse A admits in the open rooms so you could devote some time before the admissions come to be able to do that. Our team leaders work well with making sure assignments match the passion of the staff."

Published by Sciedu Press

\subsection{Call us nurse champions}

Rather than providing incentives, clinical nurses engaged in research prefer to be recognized as clinical champions. The nurses define a clinical champion as a nurse most interested in meaningful projects rather than incentives. We asked if they preferred gift cards or participation in the study and recognition in publications.

"We don't care about being included on the IRB or formally on the study. It is good to acknowledge that we worked on it [study].”

The nurses were asked if the incentives we provided (gift certificates for each unit, which were used to incentivize nurses working on the entire unit) were effective. They responded that the nurses on the unit:

"Did not really connect the incentives to the study or that it really mattered."

"I did see the point of the staff as far as... they want to feel like, what they're doing is appreciated. I don't know what that means to all of them. It means something different to so many of 'em. I struggle with what that means to them'. But a lot of the feedback I get in general is 'I just need to feel appreciated'. So, not necessarily monetary it could be a thank you note or a guardian angel pen, or something that makes that person feel like someone noticed. And it's the pat on the back kind of feeling, at least from a lot of my staff."

The nurses summarized that being recognized as a study champion and being recognized for their contribution in papers and publications is more important than any other incentive that could be offered. One participant suggested a certificate that could be framed, or other noticeable recognition would be welcomed (like a wall of recognition).

\section{WHAT WORKED?}

The nurses were asked if the length of the study (15 months) worked for them. Because they liked the product and they liked the outcome they shared "it could have gone on forever." They added about the intervention:

"If it was something we find is not adding any value we would have ended it much sooner."

Since the study was about patient-provider communication and technology was used to assist patients with communication, we asked if changes in communication were noted from pre- to post-.

"I did see changes. I still think it depended on the nurse, their comfort, and state of education. I think some nurses got it, understood it, respected the fact that the patient is a person, what they had to say was important, versus another nurse 
not knowing, not intentionally, but thinking the patient is confused and does not understand anyway and it's not important enough for me to give them this opportunity to communicate. I find now, especially developing younger nurses, that the more time that I have coaching them in certain situations, the better they do. Which is why I advocated for an educator on my unit, so that person can go out and spend one-on-one time with these new nurses who would do well with more coaching and real-time feedback."

"The nurses who truly embraced it, brought it forward to the physician providers too so they would say, no, no wait, let me run and get the tablet so the patient can communicate better. So, the nurses who truly embraced it, it made a big difference in the entire experience. The nurses who were like, maybe I will use it and maybe I won't, liked it fine but didn't embrace the idea of it. It may have made a difference and may not have but the ones who embraced it, you could truly [hear them] say 'wait' [to retrieve and bring the communication technology to the patient] on rounds or [hear them] tell the family or patient beforehand, 'let me go get this, because rounds are going to be in an hour and I want to make sure you are ready for rounds',"

\section{WHAT WORKED? WHAT DID NOT WORK? WHAT COULD HAVE BEEN IMPROVED?}

"I think just our workflow, sometimes you had to remind [nurses] to assess a patient for the need for the device. Again, that part of thinking about the patient and thinking about their patients' needs and how important it is for the patient to communicate. I tell people that communication can decrease so many things like frustration, even getting them out of restraints. If you can get the nurse to make that connection things can be so much better. I think sometimes in our workflow we just forget to ... it would be nice if we had one of you being there every day to walk through, to round, stay present, and I just think that consistency ... cause sometimes depending on the staff to do that, to say oh, this patient would be great for the device, let me call her, they just get caught up in their day and they just don't do that. Then we come along and ask, "Hey did you think about this patient?" and they go "Ohhh, I didn't think about it. I had this to do that that to do." Could use reminders, a poster, a video, or a CHAMPION (bridge the gap between the patient and the researcher)."

"What we saw that would have made it better, which you had no control over, is more tablets. Since it is not hardwired into our brains it is easy to forget. So again, those champions and weekly check-in with champions [fidelity] that say, hey, someone give me one good story this week about someone who used it. Then, the champions will look for their weekly story because they know they will be asked. This process might keep the entire project more present in their minds. To their point, a tablet was brought to the bedside after a patient consented. Therefore, nurses did not necessarily think of the use of a tablet for communication."

\section{Collaboration With SPEECH LAN- GUAGE PATHOLOGY PROFESSIONALS}

Lastly, since patient-provider communication involves speech-language pathologists, we asked if collaboration with SLPs change.

"We are working to bring SLPs and nursing together to benefit our patients. This project needs to remain nurse-led because we are at the bedside at 2 a.m. while other departments are not. Communication is always important. Night people never see SLPs and weekend people do not see SLPS. So, unless I can drive this as a nurse, own this, and I can tell those good stories, I will remember as much as I remember the swallow study [referring to the expectation to 'ask' for a swallow study for patients who may be at risk for aspiration of food and fluids].”

Good outcomes should be followed by exposure. We would like a tablet in every unit. Note the participant meant a tablet in each unit after conclusion of the study.

\section{DisCuSSiOn AND Limitations}

An informal discussion about the primary study processes and outcomes revealed that, when approached by researchers and asked to participate in a study, nurse managers are most concerned about (a) meaningful impact for their patients, and (2) how the study will affect nurse workflow. Firstly, nurse managers are concerned about their patients and how patient involvement in a study may help or hinder the healing process. Further, we learned that huddles work for education and training because huddles are an established time for review and, therefore, there is little workflow disruption. The nurse managers clearly articulated that incentives are infrequently meaningful and, therefore, may or may not incentivize nurses to enthusiastically participate. To be clear, nurse managers instead seek recognition for their participation including recognition of their role, perhaps being named as nurse champions or potential inclusion in research dissemination. This conversation was documented when a small group of nurses gathered to discuss a specific recently concluded study so that generalizability is limited. However, it is important to learn the perspectives and views, even if only in a small group of nurse leaders. Further, the information reported herein suggests that research could be conducted to explore clinicians' views of research studies and expected protocol. 


\section{CONCLUSIONS AND NEXT STEPS FOR NURSE MANAGERS}

This paper described an informal discussion with five nurse managers who were integral to the success of a research study. Over a 15-month study, collaboration was fostered by forward-thinking nurse managers who were eager to assist patients with communication. The nurse managers indicated that they are most concerned about (a) how any research study will affect their patients, and (b) how nurse workflow may be interrupted. Additionally, pre-established meeting times (huddles) work best for education and training, and study incentives may or may not be effective. Instead, they suggest recognition as nurse champions or leaders. Further, recognition as nurse champions and explication of nursing voice could be more important. Suggested ways to use this information include, 2) active engagement in research studies conducted on nursing units, 2) participation in all research activities, including dissemination of findings, 3) communication with key research personnel about anticipated time requirements for research protocol, 4) ongoing discussion about how a research protocol may affect patients and nurse workflow, and 5) suggestions for ways to recognize, support, and encourage nurses who participate in research. Nurses in this report suggest, Nurse Champions.

\section{ACKNOWLEDGEMENTS}

Thank you to all the UT Medical Center nursing units that participated in the study. The five intensive care units and nurse leaders involved in the primary study included: Trauma Surgical ICU - Theresa Day, MSN, RN, CCRN;

Progressive Care Unit - Clay Kyle, MSN, RN, PCCN; Medical Critical Care - Whitney Pickel, MSN, RN; Cardiovascular Intensive Care Unit - Kristi Boggess, MSN, RN, NE-BC and Heather Hiltbold, BSN, RN, CCRN; Neuro Critical Care - Bill Shingler, MSN, RN.

\section{FUNDING}

Partially funded by Simple Speech Synthesis (S3): Advanced Development of an mHealth Development Tool for Patients Unable to Verbalize Their Needs at the Bedside. University of Tennessee, Knoxville, College of Nursing, Office of Research Services $(\$ 9,776)$.

\section{CONFLICTS OF INTEREST Disclosure}

\section{REFERENCES}

[1] Conn VS, McCarthy AM, Cohen MZ, et al. Pearls and pitfalls of team science. West J Nurs Res. 2019; 41(6): 920-940. PMid: 30089443. https://doi.org/10.1177/0193945918793097

[2] Forbes TH, Larson K, Scott ES, et al. Getting work done: a grounded theory study of resident physician value of nursing communication. Journal of Interprofessional Care. 2020; 34(2): 225-232. PMid: 31381472. https://doi.org/10.1080/13561820.2019.1631 764

[3] Koszalinski RS, Heidel RE, Hutson SP, et al. The use of communication technology to affect patient outcomes in the intensive care unit. Comput Nurs.

[4] Koszalinski RS, Heidel R E, McCarthy J. Difficulty envisioning a positive future: secondary analyses in patients in intensive care who are communication vulnerable. Nursing \& Health Sciences. Ahead of print. November 18, 2019. PMid: 31736225. https: //doi.org/10.1111/nhs.12664

[5] Barrow MJ, Gasquoine SE. Encouraging interprofessional collaboration: The effects of clinical protocols. Journal of Clinical Nursing 2018; 27(19-20): 3482-3489. PMid: 29964305. https://doi.or $\mathrm{g} / 10.1111 /$ jocn. 14591

[6] Morrill D. Teaching teamwork and collaboration: a task-centered clinical design. Nurse Education Today. 2018; 69: 120-121. PMid: 30036710. https://doi.org/10.1016/j.nedt. 2018.07.009

[7] Berger J, Polivka B. Advancing nursing research in hospitals through collaboration, empowerment, and mentoring. J Nurs Admin. 2015; 45(12): 600-605. PMid: 26565639. https://doi.org/10.1097/ NNA. 0000000000000272 